\title{
Severe bursitis in shoulder arthritis - a manifestation of hydroxyapatite crystal deposition disease
}

\author{
Amalia Kerl-Skurka ${ }^{1}$, Beat Flueckiger ${ }^{1}$, Sabri Ali ${ }^{2}$, Martin Kilgus ${ }^{3}$, Robert Theiler $^{1}$ \\ 1. Department of Rheumatology, City Hospital Triemli, Zurich, Switzerland. 2. Department of Pathology, City Hospital \\ Triemli, Zurich, Switzerland. 3. Department of Surgery, City Hospital Triemli, Zurich, Switzerland.
}

Correspondence: Amalia Kerl-Skurka. Address: Department of Rheumatology, City Hospital Triemli, Zurich, Switzerland. E-mail: amalia.kerl-skurka@gmx.ch

Received: October 7, 2014

Accepted: November 25, 2014 Online Published: December 05, 2014

DOI : $10.5430 /$ crcp.v2n2p7

URL: http://dx.doi.org/10.5430/crcp.v2n2p7

\begin{abstract}
In the present understanding of joint pathology, the exact pathogenic role of hydroxyapatite crystals, both periarticular and intraarticular, is not yet completely clear. An association between rapidly destructive osteoarthritis (OA) and hydroxyapatite or basic calcium crystals was confirmed. This is a case report of a 78-year old female patient presenting with a five-month history of shoulder pain with swelling of both AC joints. Imaging showed a large bursitis starting in the AC joints of both shoulders. Synovial fluid analysis showed many hydroxyapatite crystals and no calcium pyrophosphate or sodium urate crystals, which confirmed the diagnosis of crystal-induced periarthropathy and AC arthritis. These periarticular radiographic and synovial fluid findings are a frequent and known manifestation of hydroxyapatite crystal deposition disease, similar to the intra-articular destructive "Milwaukee shoulder syndrome". Surgical intervention was the only therapeutic option to reduce the patient's shoulder pain.
\end{abstract}

\section{Key words}

Hydroxyapatite crystals, Bursitis, Periarticular destructive shoulder osteoarthritis, Synovial fluid

\section{I ntroduction}

For demographic reasons, more and more elderly people with chronic renal disease, metabolic disorders and polymedications are being seen with musculoskeletal and joint problems. Crystal-induced peri- and/or intra-articular arthropathy is the first and most frequent differential diagnosis of arthritis including autoimmune diseases such as rheumatoid arthritis, bursitis and rapidly progressive osteoarthritis $(\mathrm{OA})$ in elderly patients. A calcium-containing mineral (calcium hydroxyapatite) is found in bone, in soft tissue with tendon calcification and in many forms of OA. Identification of hydroxyapatite crystals is difficult and frequently is noted as an incidental finding. The most common site of calcium deposition is in the shoulder, usually in the supraspinatus tendon and bursa.

\section{Case presentation}

This is a case report of a 78-year old female patient, who within the last five months was unable to elevate her arms, due to pain and swelling of the shoulders. Up to this time, the patient had been treated for metabolic syndrome with hypertension, 
diabetes mellitus, obesity, hypertensive heart disease and polyarticular OA. She required acetylsalicylic acid, enalapril, amlodipine and metformin as medication. This patient was treated incorrectly for polymyalgia rheumatica more than ten years ago, with prednisolone $5 \mathrm{mg}$ per day taken for years combined with calcium and vitamin D supplementation.

Clinically, the patient presented with a large, 10 centimeter swollen bursa over both acromioclavicular (AC) joints with effusion of the shoulders and atrophy of the rotator cuff (see Figure 1). Passive and active elevation over $45^{\circ}$ was not possible. Radiographic imaging showed severe OA of both shoulders (see Figure 2). CT scan confirmed the severe OA and showed rotator cuff rupture, subchondral sclerosis and a large soft tissue swelling emerging from the AC joint. The effusion fluid drained from the left-sided bursa pre- and intraoperatively ( $80 \mathrm{ml}$ synovial fluid) was examined and showed 3,000 cells $/ \mu 1$ and a large quantity of hydroxyapatite crystals (see Figure 3).

Figure 1. Clinical appearance of the left shoulder (anterior view)

Figure 2. X-ray of left shoulder: glenohumeral and acromioclavicular osteoarthritis, large soft tissue swelling above the $\mathrm{AC}$ joint

Figure 3. Synovial fluid analysis of the AC bursa: clumps of hydroxyapatite crystals (alizarin red staining)
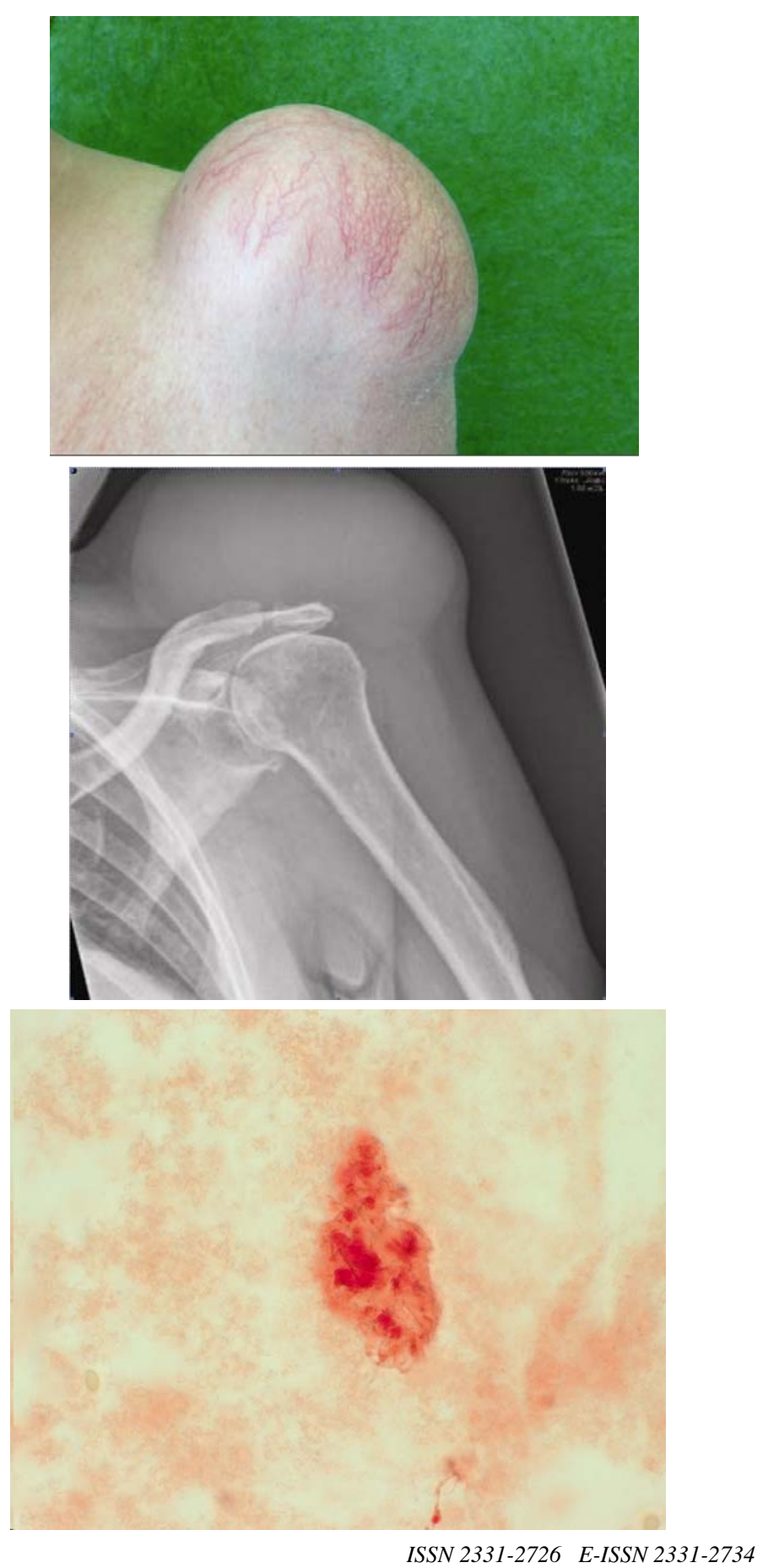
Histological examination of the left bursa showed chronic fibrous and partly fibrinous inflammation, calcification and crystals (see Figure 4-6). Bacterial culture of a blood sample and synovial fluid analysis were negative. In summary, the severe bursitis and progressive osteoarthritis of both shoulders were diagnosed as due to crystal-induced arthritis, mainly consisting of hydroxyapatite crystals, arising from the AC joints. Arthritis due to infection, gout or a neurologic pathogenesis (e.g. diabetes mellitus) and other signs of autoimmune disease such as rheumatoid arthritis were ruled out. Open bursectomy of the left shoulder confirmed a large bursa arising from the AC joint (see Figure 7). After surgery the shoulder pain was reduced, but the range of motion did not improve. Surgical intervention was the only therapeutic option to reduce the patient's shoulder pain.

Figure 4. Histological examination of the left AC bursa: chronic fibrous and partly fibrinous inflammation (HE staining)

Figure 5. Histological examination of the left AC bursa: chronic fibrous and partly fibrinous inflammation (EvG staining)

Figure 6. Histological evidence of calcification and crystals (uric acid staining)
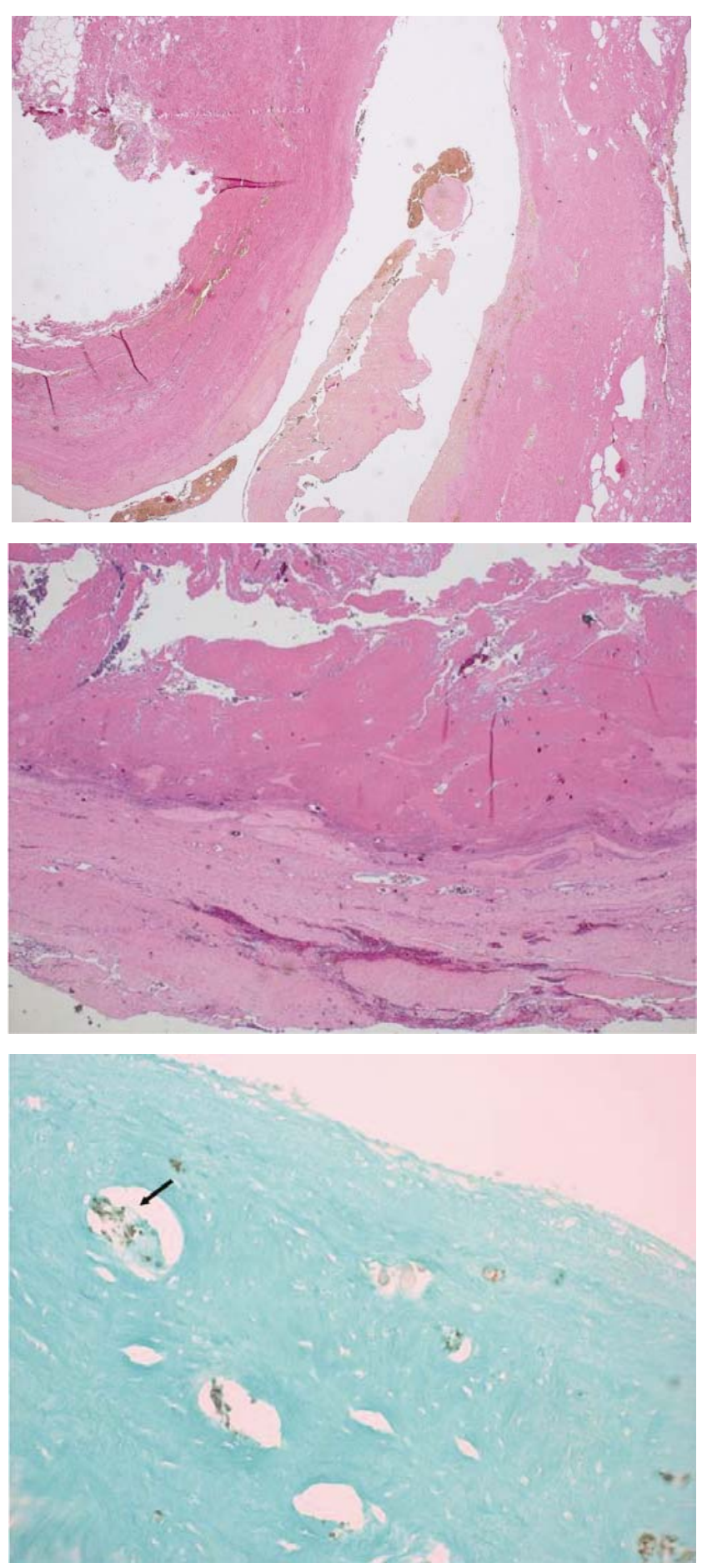
Figure 7. Open bursectomy of the left shoulder (intraoperative view)

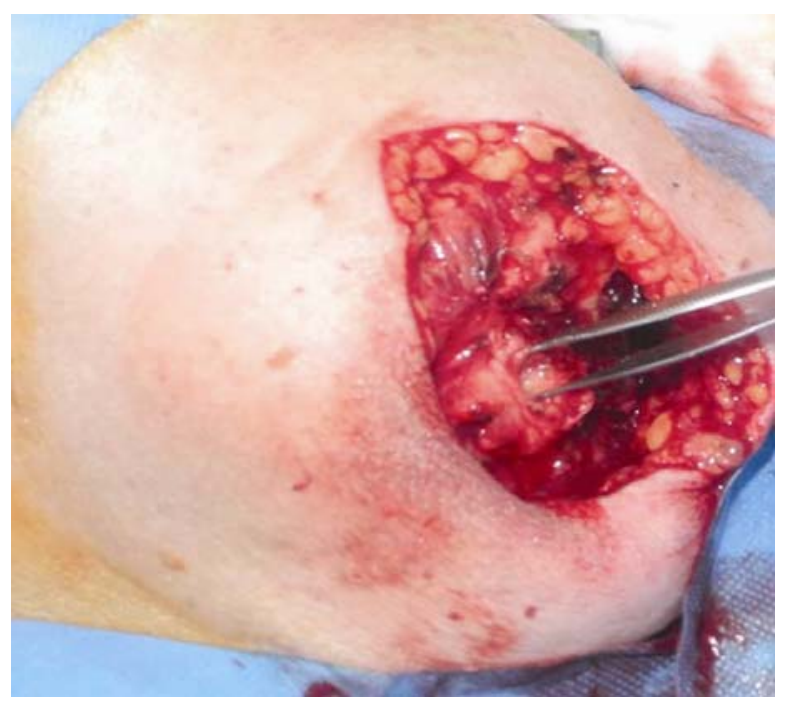

\section{Discussion}

Crystal diseases such as gout (monosodium urate crystals) and calcium pyrophosphate dehydrate (CPPD) or hydroxyapatite deposition diseases are increasingly common ${ }^{[1,2]}$. Calcium hydroxyapatite is a calcium-containing mineral that is found in bone, in soft tissue with tendon calcification and in many forms of OA. The most common site of deposition is in the shoulder, usually in the suprapinatus tendon and bursa. Hydroxyapatite diseases can affect all the joints, mainly large joints such as the knees and shoulders, but also small joints including those of the hand, wrist and foot. They can cause intra-articular and periarticular destruction but also induce chronic periarticular inflammation with bursitis and tendinitis ${ }^{[3-5]}$. The clinical picture is of an intense local inflammatory reaction to the crystals, i.e., acute arthritis with warmth, pain, swelling and loss of motion. Identification of hydroxyapatite crystals and other calcium-containing mineral referred to as basic calcium phosphate (BCP) is difficult and frequently is noted as an incidental finding. If suspected, the diagnosis is confirmed by the finding of crystals. For intra-articular detection arthrocentesis and light microscopic synovial fluid analysis are performed.

BCP often remain undetected by routine synovial fluid analysis, since their submicron size makes recognition difficult ${ }^{[6]}$. A review by Yavorskyy et al. ${ }^{[7]}$ presents an overview of the most important analytical tools used in the detection of basic calcium phosphate crystals. An ordinary light microscope has a limited resolution of about $1 \mu \mathrm{m}$ and cannot confirm basic calcium phosphate crystals, unless they aggregate into large clumps, which allows them occasionally to be observed as refractile "shiny coins". Compensated polarized light microscopy is a version of light microscopy that allows the identification of crystals by determination of the sign of their birefringence based on a color change with rotation. Basic calcium phosphate crystals usually appear as an amorphous substance, which is not birefringent and cannot be seen under polarized light microscopy. The alizarin red $\mathrm{S}$ stain is an effective method to improve the detection of hydroxyapatite crystal clumps with high sensitivity but low specificity. This was recently reported in a comprehensive review by Paul McMullan of the detection of $\mathrm{BCP}$ crystals in $\mathrm{OA}{ }^{[8]}$. Precise crystal identification such as transmission electron microscopy and more advanced microscopic techniques such as scanning electron microscopy and atomic force microscopy are expensive and not practical for the clinician. The direct effect of apatite crystals in the pathogenesis of OA was described by Hang-Korng Ea et al. ${ }^{[9,10]}$. Inflammation and cellular proliferation as well as proto-oncogene stimulation play an important role in the pathology of basic calcium phosphate crystals in degenerative arthropathies. Cartilage calcification in OA is an active process and the phenomenon is referred to as "microcrystal-induced stress" (mechanical, oxidative or cytokine stress). This is among the earliest in vivo evidence of joint destruction by basic calcium phosphate crystals. 
With advanced degenerative changes, if a large effusion is present, repeated arthrocentesis or surgical debridement may be necessary. At the same time physical therapy to maintain range of motion and muscle strength is very important, together with nonsteroidal anti-inflammatory drug treatment.

\section{References}

[1] Lawrence R, Felson DT, Helmick CG, Arnold LM, Choi H, Deyo RA, et al. Estimates of the prevalence of arthritis and other rheumatic conditions in the United States. Part II. Arthritis Rheum. 2008; 58: 26-35. PMid: 18163497. http://dx.doi.org/10.1002/art.23176

[2] Erismann M, Theiler R. Rapidly progressive osteoarthritis of the knee associated with hydroxyapatite crystals. Schweiz Med Forum. 2010; 10: 824-825.

[3] McCarty DJ, Halverson PB, Carrera GF, Brewer BJ, Kozin F. Milwaukee shoulder - association of microspheroids containing hydroxyapatite crystals, active collagenase, and neutral protease with rotator cuff defect. I. Clinical aspects. Arthritis Rheum. 1981; 24: 464-73. PMid: 6260120. http://dx.doi.org/10.1002/art.1780240303

[4] Schumacher HR Jr. Osteoarthritis and crystal deposition diseases. Curr Opin Rheumatol. 1998; 10: 244-5. http://dx.doi.org/10.1097/00002281-199805000-00014

[5] Nalbant S, Martinez JA, Kitumnuaypong T, Clayburne G, Sieck M, Schumacher HR Jr. Synovial fluid features and their relations to osteoarthritis severity: new findings from sequential studies. Osteoarthritis Cartilage. 2003; 11: 50-4. PMid: 12505487. http://dx.doi.org/10.1053/joca.2002.0861

[6] Swan A, Chapman B, Heap P, Seward H, Dieppe P. Submicroscopic crystals in osteoarthritic synovial fluids. Ann Rheum Dis. 1994; 53: 467-70. PMid: 7944620. http://dx.doi.org/10.1136/ard.53.7.467

[7] Yavorskyy A, Hernandez-Santana A, McCarthy G, McMahon G. Detection of calcium phosphate crystals in the joint fluid of patients with osteoarthritis - analytical approaches and challenges. Analyst. 2008; 133: 302-18. PMid: 18299743. http://dx.doi.org/10.1039/b716791a

[8] MacMullan P, McMahon G, McCarthy G. Detection of basic calcium phosphate crystals in osteoarthritis. Joint Bone Spine. 2011; 78: 358-63. PMid: 21273110. http://dx.doi.org/10.1016/j.jbspin.2010.10.008

[9] Ea HK, Nguyen C, Bazin D, Bianchi A, Guicheux J, Reboul P, et al. Articular cartilage calcification in osteoarthritis: insight into crystal-induced stress. Arthritis Rheum. 2011; 63: 10-8. PMid: 20862682. http://dx.doi.org/10.1002/art.27761

[10] Ea HK, Chobaz V, Nguyen C, Nasi S, van Lent P, Daudon M, et al. Pathogenic role of basic calcium phosphate crystals in destructive arthopathies. PLoS One. 2013; 8: e57352. PMid: 23468973. http://dx.doi.org/10.1371/journal.pone.0057352 